\title{
New knowledge of osteoarthrosis
}

\author{
S. YOUSUF ALI
}

\begin{abstract}
From the Experimental Pathology Unit, Institute of Orthopaedics (University of London), Royal National Orthopaedic Hospital, Stanmore, Middlesex
\end{abstract}

It is being gradually accepted that osteoarthrosis should be regarded in functional terms and, for investigative purposes, as 'joint failure'. Various anatomical, metabolic, and tissue factors can all produce the same end result in the final common pathway of cartilage degeneration, pain, and loss of joint function. Thus osteoarthrosis is a heterogeneous disease that is age-related but is not merely a consequence of ageing. This makes finding a single initial pathogenic mechanism difficult and may imply the coexistence of more than one aetiological factor. There are many reviews of osteoarthrosis (Sokoloff, 1969; Howell and Bollet, 1973; Ali et al., 1974; Kempson et al., 1975; Lee et al., 1974). This brief survey is limited to examining some of the recent concepts and developments in the study of osteoarthrosis and joint failure.

Osteoarthrosis has been divided traditionally into primary and secondary forms. We can argue that with sufficient knowledge of the disease we shall be able to describe all osteoarthrosis as secondary to some initiating factor. At present there are varieties which, because we lack knowledge of a cause, we term primary or idiopathic. It is this type of disorder which needs an explanation most urgently. It is noteworthy that some varieties of secondary osteoarthrosis are due to mechanical failure or mechanical misalignment of the joints. In this category we find osteoarthrosis secondary to congenital hip dislocation, Perthes's disease, slipped capital femoral epiphysis, and other forms of osteoarthrosis due to altered geometry of the femoral head, femoral neck, and acetabular interrelationships. We are familiar, too, with some of the endocrine and metabolic influences that cause osteoarthrosis such as acromegaly, chondrocalcinosis, and ochronosis.

\section{Use and misuse of joints}

There have always been doubts about the hypothesis that the general wear and tear of joints leads to cartilage degeneration and osteoarthrosis. Experiments with excessive use of joints by long-term, forced running on a motor driven cylinder caused little degenerative change in mice (Lanier, 1946).
In Finland Puranen et al. (1975) studied the hip joints of $\mathbf{7 4}$ former runners to determine the significance of physical strain in the development of osteoarthrosis. Primary osteoarthrosis was present in only $4 \%$ of the athletes but in $8.7 \%$ of the normal controls. They thought that competitive sports could not be a factor in making osteoarthrosis more likely, nor did the joints of any of the runners have changes indicating epiphyseolysis which Murray and Duncan (1971) had found in athletes. Puranen et al. concluded that the physical strain to which runners' hips are subjected is not a cause of osteoarthrosis and that normal use of the joint is good for its health.

The extensive studies in Leeds on this subject have also cast doubt on the simple wear-andtear explanation for osteoarthrosis (Adams, 1976; Burke et al., 1977; Murray-Leslie et al., 1977). Those thought to be likely to get osteoarthrosis by virtue of their occupation or sport, such as professional footballers (Brodelius, 1961), pneumatic drillers (Radin et al., 1972), and parachutists (Jackson, 1968) have been found to have no more joint degeneration than the rest of the population. Moreover, 25 grossly obese patients showed no further osteoarthrosis than the average (Goldin et al., 1976). The only common factor among the parachutists with osteoarthrosis was meniscectomy, a well-known association (Jackson, 1968). Thus clearly normal or even excessive use of joints does not necessarily lead to osteoarthrosis.

\section{Changes on joint surface}

A very important contribution to our knowledge of osteoarthrosis and of the changes that occur in articular cartilage was made by Byers et al. (1970). They found that the most frequent change that normally occurs with age in normal femoral heads is fibrillation in various non-pressure-bearing areas. They termed this a non-progressive lesion and observed that it did not lead to osteoarthrosis. In another set of femoral heads they noticed that some lesions on the superior surface of the femoral heads were progressive; these could be correlated with osteoarthrotic changes. In this way, for the first time, fibrillation of the non-osteoarthrotic type and fibril- 
lation, in certain pressure bearing areas, of an osteoarthrotic type could be differentiated. This important contribution has facilitated a better biochemical and ultrastructural characterisation of the osteoarthrotic tissue as compared with the non-osteoarthrotic fibrillated tissue.

Byers et al. (1970) found that the progressive osteoarthrotic lesion affected about $4 \%$ of a caucasian population while the non-progressive affected a very large number which increased with age, reaching a maximum of $80 \%$ in the seventh decade for the most prevalent feature. Hoaglund et al. (1973) estimated the incidence of grade 3 and 4 osteoarthrosis of hip in Hong Kong Chinese as being only $1.2 \%$ for men and almost zero for women. A similar study found a very low incidence of osteoarthrosis in the Indian population and led to the conclusion that in these Asian populations primary osteoarthrosis is extremely rare. Byers et al. (1975) examined necropsy specimens of femoral head from Chinese and Indians and found that the number and severity of many non-progressive lesions in the Asians was less than in caucasians. Progressive changes were very rare. These geographical and ethnic differences indicated that osteoarthrosis is not due merely to ageing of the tissues. There are other factors that need further investigation.

Meachim and Emery (1973) have studied the incidence of cartilage fibrillation in various joints at necropsy in the Liverpool population, using an Indian ink technique. Their results and conclusions about the changes with age in normal articular cartilage agree largely with those of Byers et al. (1970). They show that from teenage onwards focal areas of cartilage splitting and disintegration are extremely common in adult synovial joints. These lesions tend to spread tangentially across a joint surface to affect an increasingly large area. At certain sites the lesions may progress vertically to expose bone. These sites closely resemble the progressive areas in the femoral head described by Byers $e t$ al. (1970).

These findings mean that the surface cartilage of a normal joint is not completely intact but has both intact and fibrillated areas. Therefore in considering the initiation of osteoarthrosis we should differentiate between the osteoarthritic type of fibrillation as compared with normal fibrillation. In this instance osteoarthrosis is a disease caused by at least two factors-the first general change in cartilage is accelerated by a second factor causing abnormal wear, as in the zenith of the femoral head in hip joint disease.

\section{Joint geometry and congruity}

The geometrical shape of the different components of the human joint can have a very important effect on its functional quality. Bullough et al. (1973) found that the fit or 'sphericity' of the ball-andsocket type of joint changed with age. After examining many joints they concluded that as people grow older the contours on a joint give a better fit. Joint contours that are incongruent in youth become less so with age and therefore the contact between opposing surfaces becomes greater. The effect that this change may have on the load transmitted through cartilage is very considerable in engineering terms.

Bullough et al. (1973) showed that the head of the femur 'misfits' the acetabulum in a very particular way. There is a gap on the superior surface. With advancing age the joint assumes fuller congruity and this uppermost zone gains contact with the acetabulum. This contact puts additional pressure on an area of the femoral head which to that date had not participated in articulation unless a heavy load was put on it. Individuals in whom this type of congruity is achieved early are then particularly susceptible to osteoarthrosis. This important evidence has received support from other laboratories where such phenomena have been studied and where the contact areas of various additional joints have been mapped out.

There are at least two important implications of this concept. The first is that the health, thickness, and compliance of articular cartilage itself may change the geometric configuration of a joint and therefore make the contact areas vary with age or with disease. The second is that a change in geometry may be brought about by a remodelling of the subchondral bone or in the calcified cartilage due to altered pressure (Goodfellow and Mitsou, 1977).

\section{Compliance of cartilage and subchondral bone}

The compliance and elasticity of human articular cartilage changes with age. Gardner and his colleagues (Armstrong et al., 1977) measured the deformation of femoral head cartilage under loads of up to five times body weight on 30 whole hip joints obtained post mortem, illustrating graphically the results from a young (32-year-old) and an old (80-year-old) woman. They concluded that young adult cartilage is firm and the reduction in thickness with physiological loads is very little, whereas older cartilage is much more compliant and deforms up to $15 \%$ of its thickness when an identical load is applied. The implications from this work are at least twofold. Firstly, the more compliant cartilage is likely to encounter a greater contact area of the opposing joint surface, changing both the congruity of the joint and the wear on the contact area. Secondly, a change in compliance may be brought 
about by any change in chemical constitution of the cartilage that alters its characteristic response to load.

An important role for the underlying bone in the pathophysiology of joint function has been demonstrated by the work of Radin and his colleagues (Radin, 1976). They showed that changes of a subtle and clinically undetectable nature may be at work in the subchondral bone region. They suggest that there may be an increase in the rigidity of the subchondral cancellous bone, so imparing the joints capacity to bear dynamic stress without damage to cartilage. They maintain that the nature of the mechanical stress which is most destructive to cartilage is the repetitive impulsive loading that creates tensile fatigue. This is evident with age. The subchondral bone seems to be extremely sensitive to such repetitive loading.

Radin $e t$ al. believe that increased rigidity may be caused by callus formation secondary to the isolated fatigue fractures that are seen in the subchondral bone region in the femoral head and other joints. In conformity with this view it should be remembered that Johnson (1962) had suggested remodelling of the subchondral bone region as an initiating cause in osteoarthrosis. Foss and Byers (1972) found that osteoarthrosis is commoner in people with an aboveaverage skeletal mass or bone density, implying that osteoarthrosis may be associated with rigid bones.

\section{Changes in cartilage enzymes and proteoglycans}

Although a number of these factors may operate in initiating osteoarthrosis it is generally accepted that the tissue most affected first is cartilage and that the other tissues such as the synovial membrane, subchondral bone, and joint capsule are affected later. Articular cartilage has therefore been subjected to detailed ultrastructural, biomechanical, and biochemical studies.

Articular cartilage derives its characteristic resilience and weight-bearing properties from the interaction of its chemical constituents. Collagen, proteoglycans, and other minor constituents such a glycoproteins and mineral are the most important components of cartilage. The way water is bound to some of these polymers determines how the tissue is going to react to pressure. The role of proteoglycans in providing cartilage with many of its characteristic properties is now well established (Ali et al., 1974; Freeman, 1973; Muir, 1977). Proteoglycans are lost from articular cartilage matrix in osteoarthrosis and the chondrocytes in osteoarthrotic cartilage are active in the synthesis of proteoglycans, with a high turnover (Mankin, 1974; Maroudas, 1975).

The dramatic effect of proteolytic enzymes on the degradation of cartilage in vivo and of various similar agents in producing osteoarthrosis in animals has led to the suggestion that there may be potent enzymes present in cartilage which bring about its rapid breakdown by upsetting the equilibrium between the anabolic and catabolic activity of the tissue. Systematic investigations of the endogenous enzymes present in cartilage that may cause degradation of the matrix from within under abnormal conditions have been made in several laboratories (Ali, 1964; Chrisman, 1969; Dingle, 1973; Howell and Bollet, 1973). Our studies with cartilage, obtained from various sites and species, have established the spectrum of cathepsins present in cartilage and capable of degrading proteoglycan molecules (Ali and Evans, 1973). The level of degradative enzymes in human osteoarthrotic articular cartilage is raised, which may explain the loss of proteoglycans from diseased tissue. Results have been summarised in detail elsewhere (Ali and Bayliss, 1974), but it can be seen from the Table that cathepsin $\mathrm{D}$ activity in osteoarthrotic tissue is almost twice that of normal articular cartilage. We have also found that there is a change in the polymeric structure of the proteoglycans in human arthrotic tissue (Bayliss and Ali, 1978). These analyses have shown that the concentrations of degradative enzymes in human osteoarthrotic tissue are raised and the proteoglycans altered.

This change in the nature and level of proteoglycan in articular cartilage can have several consequences. Freeman (1972) and Kempson (1975) showed that the compressive stiffness of articular cartilage is dependent on its proteoglycan content. Depletion of proteoglycans would make it much more susceptible to degeneration. Moreover, proteoglycans in cartilage have been variously shown to participate in the water binding of the tissue, the type of collagen fibrils formed, calcification of the matrix, and even the prevention or control of vascularity and capillary formation. Loss of proteoglycans from articular cartilage will affect some of these factors and alter the characteristics of cartilage in such a way that it

Table Comparison of enzyme activity in normal and osteoarthrotic human articular cartilage (50-80 age group). Mean values are given and numbers in parentheses denote number of specimens analysed. (For further details see Ali and Bayliss, 1974)

\begin{tabular}{lll}
\hline Enzyme & \multicolumn{2}{l}{ Type of cartilage } \\
\cline { 2 - 3 } & Normal & Osteoarthrotic \\
\hline $\begin{array}{c}\text { Cathepsin D (mg tyrosine/g } \\
\text { cartilage) }\end{array}$ & $0.560(28)$ & $0.930(34)$ \\
$\begin{array}{c}\text { Alkaline phosphatase (mg } \\
\text { phosphate/g cartilage) }\end{array}$ & $0.038(19)$ & $0.431(23)$ \\
\hline
\end{tabular}


becomes much more susceptible to rapid physical destruction during normal joint movement in some osteoarthrotic patients.

\section{Changes in collagen}

Collagen is the major polymeric component of articular cartilage and the network of its fibres retains the characteristic proteoglycan gel. Water is trapped within the gel. The tensile properties of human adult articular cartilage are due mainly to the collagen fibre network. These have been extensively studied by Freeman (1972) and Kempson (1975). Kempson (1973) has shown that the tensile properties vary from the surface through the deeper layers and that this variation can be correlated with the variation in the collagen composition of cartilage. He found that the tensile strength and stiffness of articular cartilage change with increasing age. This change takes the form of diminishing strength and diminishing stiffness. Since the occurrence of osteoarthrosis and the incidence of fibrillation both increase with increasing age this loss of tensile strength may be related to the genesis of osteoarthrosis. Weightman (1975) has shown, firstly, that human adult articular cartilage in tension is indeed fatigue prone and, secondly, that the fall in fatigue resistance with age is so pronounced that tissue from cadavers of 20 years of age would not be expected to fail after the application of over 100 million cycles at a stress of $5 \mathrm{MN} / \mathrm{m}^{2}$, whereas this stress would produce failure in the tissue of cadavers of 50 years of age after application of the order of only 10 million cycles. Weightman (1975) has also shown that a cyclically applied compressive load produces fragmentation of the surface of loaded articular cartilage and an appearance very similar to the fibrillation seen in human joints.

Continuing with his search for a primary aetiological factor in osteoarthrosis, Freeman and colleagues (Day et al., 1975) identified an anatomical feature in the acetabulum which makes contact at low loads with the zenith zone of the femoral head. This feature produces a contact pressure area on the zenith of the femoral head corresponding to the congruity observed by Bullough et al. (1973). On the basis of these experiments Freeman (1975) has advanced the hypotheses that in some cases of osteoarthrosis fibrillation may be due to collagen fatigue failure in the articular cartilage and that this phenomenon is age-related (because the fatigue resistance of the collagen network in articular cartilage diminishes sharply with increasing age). The fall in the tensile stiffness and strength of articular cartilage is compatible with this view. The evidence suggests that although the amount of collagen in the matrix of cartilage does not diminish with increasing age, or with the onset of osteoarthrosis, the mechanical integrity of the network is prone to failure. This implies that the collagen fibres, or the cross-links between them, are breaking. Thus, once the surface has become fragmented, the collagen network which normally retains the proteoglycans becomes disrupted, releasing the proteoglycans into the joint This secondary leakage is consequential on the loosening of the collagen network. Freeman (1975) goes on to suggest that the particular triangular fibrocartilagenous area at the zenith of the acetabulum may produce greater contact pressure than normal. This anatomical feature may be much more common in certain populations than others, producing osteoarthrosis more often in caucasians than in Asians.

\section{Remodelling and abnormal mineralisation}

Observations by Johnson $(1959,1962)$ led him to the idea of continuous remodelling of subchondral bone as a general concept, not confined to the temporomandibular joint of human adults. In a study of adult human patellae, Green et al. (1970) concluded that there is continuous growth activity in the osteochondral region during the adult years. The work of Radin (1976) persuaded him that changes first occurred in the subchondral plate which made cartilage much more susceptible to degeneration. All these studies have been on osteoarthrotic material; it was not easy to determine whether the changes in the subchondral bone and the remodelling process preceded or succeeded the osteoarthrotic process. From observations by surgeons, who noticed the general absence of osteoarthrosis in the head of femurs excised in the treatment of fractures of the femoral neck, Ball (1966) first confirmed the impression that osteoporotic patients did not develop osteoarthrosis. This stimulated Foss and Byers (1972) to study the metacarpal bone density of osteoarthrotic patients and compare it with normals. They found that the metacarpal bone density of most patients with osteoarthrosis of the hip joint was greater than normal for their age. They confirmed the association between fracture of the upper end of the femur and osteoporosis, and showed that osteoarthrosis of the hip is seldom found in such patients.

From our biochemical and enzymatic analyses of human osteoarthrotic cartilage (Ali and Evans, 1973) we postulated a calcification abnormality in diseased tissue as one aetiological factor. Electron microscopy observations of diseased human articular cartilage also demonstrated amorphous and crystalline mineral deposits in cartilage matrix (Ali and Bayliss, 
1974; Ali and Wisby, 1975). Because McCarty (1970) had recognised apatite among the variety of crystals seen in human articular and periarticular structures in inflammatory arthritis and apatite nodules had been found in the synovial fluid of arthritic patients (Dieppe et al., 1976) we made a further search for the mechanism of apatite crystal formation in human articular cartilage. Two recent papers detail our findings on the subject of apatite crystal formation in normal and osteoarthrotic cartilage (Ali, 1977; Ali, 1978). Here we summarise the evidence for the formation of mineral nodules from matrix vesicles and outline the concept of abnormal mineralisation of articular cartilage as one process which could initiate osteoarthrosis.

After the closure of the epiphyseal plate the articular cartilage normally remains protected from further calcification. This allows it to remain resilient and to perform its weight-bearing function satisfactorily. Our studies have led us to believe that it is perhaps more relevant to regard articular cartilage, especially near the osteochondral junction, as a latent growth plate that can change under various conditions and revert back to a growth phase, again behaving like an epiphyseal plate. Our investigations of epiphyseal plate calcification had shown that the first needles of apatite are formed in matrix vesicles by calcium incorporation. The crystal nodules thus formed accrete more mineral and coalesce to form the calcified septa (Ali et al., 1970; Ali, 1976). The systematic mineralisation of the growth plate is therefore initiated by the matrix vesicles, which are extracellular, membranous particles about $100 \mathrm{~nm}$ in diameter containing most of the alkaline phosphatase, pyrophosphatase, and ATPase activity of the tissue (Ali et al., 1970).

With this knowledge of matrix vesicle function in growth cartilage we decided to look at human articular cartilage to see if the vesicles play a part in the mineralisation of joint tissue, especially under pathological conditions. Ultrastructural examination of normal articular cartilage confirmed that the dense bodies surrounding the pericellular area, which had been described by various other authors as lipid globules, were matrix vesicles similar to those in epiphyseal cartilage. Their appearance showed the same osmiophilic characteristics; their size varied from $50 \mathrm{~nm}$ to $250 \mathrm{~nm}$ in diameter and was very similar to the matrix vesicles in epiphyseal cartilage. However, they appeared more heterogeneous in size and distribution (Fig. 1). The vesicles were present at all levels of articular cartilage but were more often in the deeper zone near the tide mark region adjoining the subchondral bone. In this region microcrystals were present inside the vesicles and mineral nodules could be seen either forming from vesicles or lying next to them(Fig. 1). Obviously it was impossible by electron microscopy to determine their frequency and occurrence. We had to rely on the amount of alkaline phosphatase activity as a means of quantification.

In other published work we have described the increased alkaline phosphatase activity in osteoarthrotic cartilage (Table). This was sometimes up to 30 times higher than that of normal articular cartilage in the same age group (Ali and Evans, 1973; Ali and Bayliss, 1974; Ali, 1977). This may indicate that the number of matrix vesicles in osteoarthrotic cartilage is increas_d. Ultrastructurally, osteoarthrotic articular cartilag. appiared to have many more matrix vesicles, not only in the deeper but also in the mid-zone. The vesicles also appeared to be more granular and often contained needle-like crystals. The crystals were more common in the deeper zone. (Fig. 1).

Our electron microscopy and biochemical studies indicate that the calcification mechanism may be abnormal in osteoarthrotic cartilage and that this could have serious consequences. Articular cartilage has a great affinity for calcium and a high calcium content (Eichelberger et al., 1958). An abnormal calcification mechanism can produce cartilage degeneration in a number of ways. For example, an increase in matrix vesicles and mineral nodules in the deeper zones of cartilage may lead to focal conversion of the basal portion of the tissue into calcified cartilage and eventually bone. This may alter the tide mark or osteochondral junction region and permit an advance of the mineral front, implying that articular cartilage has reverted to a growth phase again. Remodelling of the osteochondral junction may put greater pressure on the remaining layers of cartilage, which may then become more susceptible to degradation by normal wear processes as indicated by Johnson (1962) and confirmed by others (Vignon et al., 1974; Lane et al., 1977). Moreover, any change in the local concentration of calcium in articular cartilage could alter the physicochemical properties of the tissue, such as the water-binding capacity, the colloidal charge density, the swelling pressure, and thus elasticity of the tissue.

Gardner and his colleagues (Armstrong et al., 1977) have shown that articular cartilage becomes more compliant with age, and this shift could be affected not only by the concentration of proteoglycans but also the mineral content of the cartilage. All these changes will make the articular cartilage more prone to wear under pressure during normal use. In addition, the presence of mineral crystallites and nodules in the middle and upper layers of cartilage may lead to physical, abrasive wear of the whole tissue. 


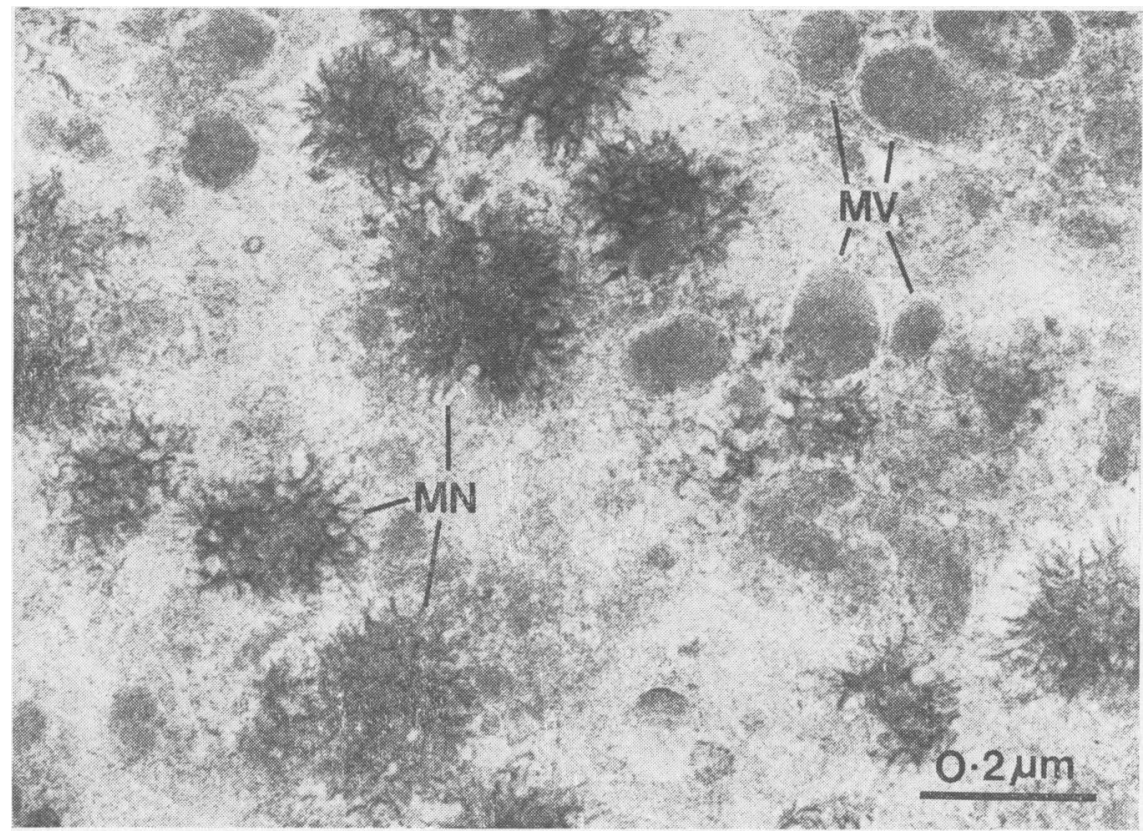

Fig. 1 Electron micrograph of matrix vesicles $(M V)$ and mineral nodules $(M N)$ in pericellular region of the deep zone of an early osteoarthrotic human (aged 32 years) articular cartilage. Note matrix vesicles closely associated with apatitelike crystal clusters and nodules. (Uranylacetate and lead citrate $\times$ $92000)$

\section{Apatite crystals and inflammation}

The results of our investigations of matrix vesicles and of apatite-like crystals and nodules in human articular cartilage can be correlated with some of the consequences of crystal-induced inflammation in joints. McCarty's (1970) concept of 'crystal deposition disease' showed that, apart from urate and pyrophosphate deposits, two other crystal types could be found in human articular cartilage and in adjoining tissue and fluid. These types were hydroxyapatite and dicalcium phosphate dihydrate.

Recurrent acute inflammation associated with focal apatite crystal deposition has been found in some patients (McCarty and Gatter, 1966). Dieppe et al. (1976) and Dieppe (1977) not only identified and analysed hydroxyapatite nodules in synovial fluid but also demonstrated the inflammatory reaction which the mineral particles could produce. Thus they postulate an 'apatite deposition disease' as one form of osteoarthrosis. The particle sizes described by Dieppe et al. (1976) vary from $0.15 \mu \mathrm{m}$ to $0 \cdot 8$ $\mu \mathrm{m}$. The matrix vesicles we found in human articular cartilage were from 0.5 to $0.25 \mu \mathrm{m}$. When nodules were formed from them the diameter increased to $0.6 \mu \mathrm{m}$ (Fig. 1). Thus the mineral nodules in Fig. 1 are very near in size and description to those of Dieppe et al. (1976).

In normal articular cartilage the mineral nodules associated with matrix vesicles are commonly found in the deeper layers. In osteoarthritic cartilage they also occur in the middle zones. If there were coincident deep fibrillation on the formation of vertical clefts in the cartilage then it would be easy to visualise these nodules being shed into the joint cavity, setting up an inflammatory cycle. In chondrocalcinosis there seems to be a slight decrease in the alkaline phosphatase activity of articular cartilage (Reginato et al., 1974). By contrast, we have found that alkaline phosphatase activity is increased in osteoarthrotic cartilage. Our findings are therefore quite different from those in pseudogout. Possibly lack of alkaline phosphatase (pyrophosphatase) will lead to an accumulation of pyrophosphate, whereas an increase in alkaline phosphatase will permit the hydrolysis of phosphate-containing substrates and the formation of apatite (Ali, 1976). We therefore agree with Dieppe et al. (1976) that apatite deposition disease should be added to the crystal deposition diseases defined by McCarty (1970). We suggest that the formation of apatite nodules in cartilage and in synovial fluid is largely dependent on the distribution and function of matrix vesicles.

\section{Conclusions}

In this brief review of newer knowledge of osteoarthrosis it has been easy to accept that anatomical, mechanical, or metabolic predisposing factors can easily lead to secondary osteoarthrosis. Where 
Predisposing factors: Anatomical, mechanical, metabolic, tissue composition

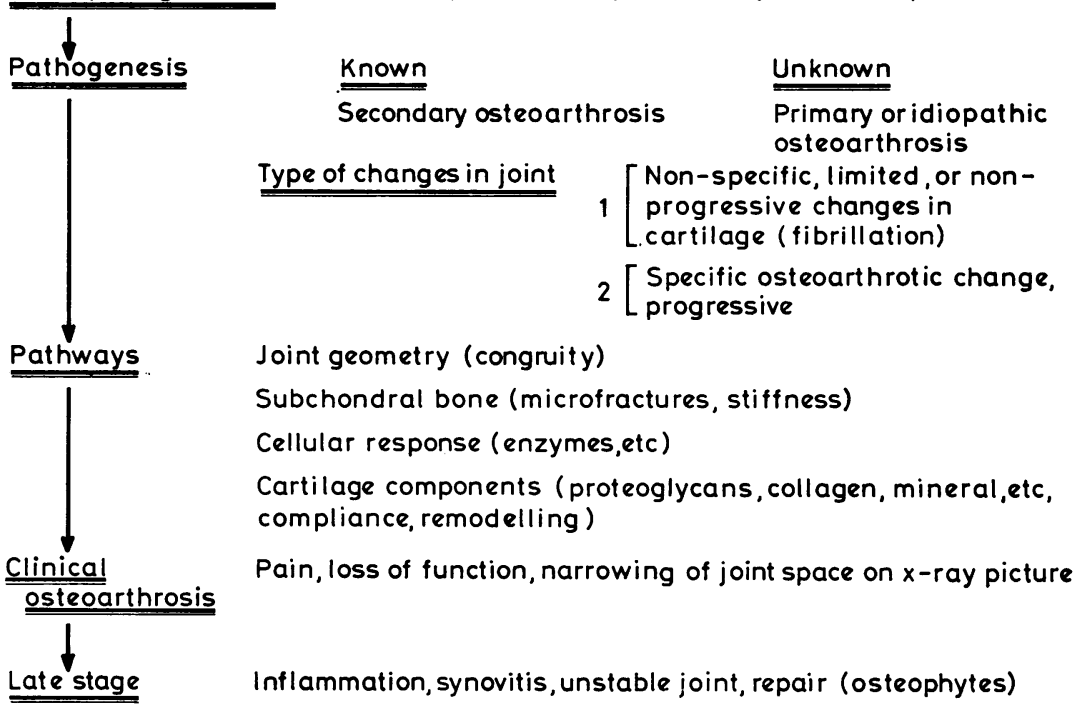

Fig. 2 Some relevant features of osteoarthrosis. primary factors in the joint tissues are concerned we are on less certain ground. Most of the current research is directed towards this end-that is, in trying to determine the role of collagen, proteoglycan, and mineral, and any change in cartilage composition that will make the tissue more susceptible to subsequent secondary mechanical degeneration. Clearly, even where these tissue components are concerned there may be different causes leading to the same long-term effect of total cartilage degeneration.

Because the initiating factors are various and the disease heterogeneous it is difficult to imagine that we shall achieve a full understanding or a simple cure in the near future. Some of the important and relevant features are outlined in Fig. 2. A number of other important components are omitted owing to lack of space. The points mentioned are merely pieces in a jigsaw. If there were complete and final agreement of the picture (disease) some sense of the odd pieces might be made. At present most research workers have their own picture of osteoarthrosis. The pieces they possess or the features they select for study cannot yet convey the full story. One thing is clear-clinical osteoarthrosis is diagnosed when the joint is near total failure and unlikely to improve. Most of the events leading to this crisis have been overtaken by numerous secondary changes; the original initiating factors have been lost. To date, there is no diagnostic test to give early warning before the onset of clinical, irreversible, osteoarthrosis. Till we understand the initiating factors or the preliminary stages and can predict the disease in advance any specific chemotherapy seems a pious hope. Meanwhile prosthetic joint replacement and other surgical intervention remain the only means of ameliorating the consequence of osteoarthrosis.

I thank Miss Lois Evans, Mrs Angela Wisby, and Dr Michael Bayliss for assistance with my research projects. I am indebted to the PF Charitable Trust and the Nuffield Foundation for financial support.

\section{References}

Adams, I. D. (1976). Osteoarthrosis and sport. Clinics in Rheumatic Diseases, 2, 523-541.

Ali, S. Y. (1964). The degradation of cartilage matrix by an intracellular protease. Biochemical Journal, 93, 611-618.

Ali, S. Y. (1976). Analysis of matrix vesicles and their role in the calcification of epiphyseal cartilage. Federation Proceedings, 35, 135-142.

Ali, S. Y. (1977). Matrix vesicles and apatite nodules in arthritic cartilage. In Perspectives in Inflammation, edited by D. A. Willoughby, J. P. Giroud, and G. P. Velo, pp. 211-223. MTP Press, Lancaster.

Ali, S. Y. (1978). Mineral-containing matrix vesicles in human osteoarthrotic cartilage. In Aetiopathogenesis of Osteoarthrosis, edited by G. Nuki. Pitman Medical, London. (In press.)

Ali, S. Y., and Bayliss, M. T. (1974). Enzymic changes in human osteoarthrotic cartilage. In Normal and Osteoarthrotic Articular Cartilage, edited by S. Y. Ali, M. W. Elves, and D. H. Leaback, pp. 189-202. Institute of Orthopaedics, London.

Ali, S. Y., Elves, M. W., and Leaback, D. H. (1974). Normal and Osteoarthrotic Articular Cartilage. Institute 
of Orthopaedics, London.

Ali, S. Y., and Evans, L. (1973). Enzymatic degradation of cartilage in osteoarthritis. Federation Proceedings, 32, 1494-1498.

Ali, S. Y., Sajdera, S. W., and Anderson, H. C. (1970). Isolation and characterisation of calcifying matrix vesicles from epiphyseal cartilage. Proceedings of the National Academy of Sciences of the United States of America, 67, 1513-1520.

Ali, S. Y., and Wisby, A. (1975). Ultrastructural aspects of normal and osteoarthrotic cartilage. Annals of the Rheumatic Diseases, 34, Supp. 2, 21-23.

Armstrong, C. G., Bahrani, A. S., and Gardner, D. L. (1977). Alteration with age in compliance of human femoral-head cartilage. Lancet, 1, 1103-1104.

Ball, J. (1966). Personal communication quoted by Byers et al. (1970).

Bayliss, M. T., and Ali, S. Y. (1978). Isolation of proteoglycans from human articular cartilage. Biochemical Journal, 169, 123-132.

Brodelius, A. (1961). Osteoarthrosis of the talar joints in footballers and ballet dancers. Acta Orthopaedica Scandinavica, 30, 309-314.

Bullough, P., Goodfellow, J., and O'Connor, J. (1973). The relationship between degenerative changes and load-bearing in the human hip. Journal of Bone and Joint Surgery, 55B, 746-758.

Burke, M. J., Fear, E. C., and Wright, V. (1977). Bone and joint changes in pneumatic drillers. Annals of the Rheumatic Diseases, 36, 276-279.

Byers, P. D., Contepomi, C. A., and Farkas, T. A. (1970). A post-mortem study of the hip joint. Including the prevalence of the features of the right side. Annals of the Rheumatic Diseases, 29, 15-31.

Byers, P. D., Hoaglund, F. T., Purewal, G. S., and Yau, A. C. M. C. (1975). Articular cartilage changes in Caucasian and Asian hip joints. Annals of the Rheumatic Diseases, 33, 157-161.

Chrisman, O. D. (1969). Biochemical aspects of degenerative joint disease. Clinical Orthopaedics, 64, 77-86.

Day, W. H., Swanson, S. A. V., and Freeman, M. A. R. (1975). Contact pressures in the loaded human cadaver hip. Journal of Bone and Joint Surgery, 57B, 302-313.

Dieppe, P. A. (1977). Crystal-induced inflammation and osteoarthritis. In Perspectives in Inflammation, edited by D. A. Willoughby, J. P. Giroud, and G. P. Velo, pp. 225-235. MTP Press, Lancaster.

Dieppe, P. A., Huskisson, E. C., Crocker, P., and Willoughby, D. A. (1976). Apatite deposition disease, a new arthropathy. Lancet, 1, 266-268.

Dingle, J. T. (1973). The role of lysosomal enzymes in skeletal tissues. Journal of Bone and Joint Surgery, 55B, 87-95.

Eichelberger, L., Akeson, W. H., and Roma, M. (1958). Biochemical studies of articular cartilage, I. Normal Values. Journal of Bone and Joint Surgery, 40A, 142152.

Foss, M. V. L., and Byers, P. D. (1972). Bone density, osteoarthrosis of the hip, and fracture of the upper end of the femur. Annals of the Rheumatic Diseases, 31, 259-264.

Freeman, M. A. R. (1972). The pathogenesis of primary osteoarthrosis: an hypothesis. Modern Trends in Orthopaedics, 6, 40-94.

Freeman, M. A. R. (1973). Adult Articular Cartilage. Pitman Medical, London.

Freeman, M. A. R. (1975). The fatigue of cartilage in the pathogenesis of Osteoarthrosis. Acta Orthopaedica Scandinavica, 46, 323-328.

Goldin, R. H., McAdam, L., Louie, J. S., Gold, R., and Bluestone, R. (1976). Clinical and radiological survey of the incidence of Osteoarthrosis among obese patients. Annals of the Rheumatic Diseases, 35, 349353.

Goodfellow, J., and Mitsou, A. (1977). Joint surface incongruity and its maintenance. An experimental study. Journal of Bone and Joint Surgery, 59B, 446-451.

Green, W. T., jr, Martin, G. N., Eanes, E. D., and Sokoloff, L. (1970). Microradiographic study of the calcified layer of articular cartilage. Archives of Patho$\log y$, 90, 151-158.

Hoaglund, F. T., Yau, A. C. M. C., and Wong, W. L. (1973). Osteoarthritis of the hip and other joints in Southern Chinese in Hong Kong. Journal of Bone and Joint Surgery, 55A, 545-557.

Howell, D. S., and Bollet, A. J. (1973). Workshop on osteoarthrosis. Federation Proceedings, 32, 1457-1506.

Jackson, J. P. (1968). Degenerative changes in the knee after meniscectomy. British Medical Journal, 2, 525-527.

Johnson, L. C. (1959). Kinetics of osteoarthritis. Laboratory Investigation, 8, 1223-1238.

Johnson, L. C. (1962). Joint remodelling as a basis for osteoarthritis. Journal of the American Veterinary Medical Association, 141, 1237-1241.

Kempson, G. E. (1973). The mechanical properties of articular cartilage. In Adult Articular Cartilage, edited by M. A. R. Freeman, pp. 171-227. Pitman Medical, London.

Kempson, G. E. (1975). Mechanical properties of articular cartilage and their relationship to matrix degradation and age. Annals of the Rheumatic Diseases, 34, Supp. 2, 111-113.

Kempson, G. E., Maroudas, A., and Weightman, B., eds (1975). Conference on articular cartilage. Annals of the Rheumatic Diseases, 34, Supp. 2, 1-159.

Lane, L. B., Villacin, A., and Bullough, P. G. (1977). The vascularity and remodelling of subchondral bone and calcified cartilage in adult human femoral and humeral heads. Journal of Bone and Joint Surgery, 59B, 272-278.

Lanier, R. R. (1946). The effects of excercise on the kneejoints of inbred mice. Anatomical Record, 94, 311-321.

Lee, P., Rooney, P. J., Sturrock, R. D., Kennedy, A. C., and Dick, W. C. (1974). The etiology and pathogenesis of osteoarthrosis: A review. Seminars in Arthritis and Rheumatism, 3, 189-218.

Mankin, H. J. (1974). Biochemical abnormalities in articular cartilage in osteoarthritis. In Normal and Osteoarthrotic Articular Cartilage, edited by S. Y. Ali, M. W. Elves, and D. H. Leaback, pp. 153-171. Institute of Orthopaedics, London.

Maroudas, A. (1975). Glycosaminoglycan turn-over in articular cartilage. Philosophical Transactions of the Royal Society, London. Series B. Bilogical Sciences, 
271, 293-313.

McCarty, D. J. (1970). On the Crystal Deposition Diseases. DM: Disease-a-month, 3-31.

McCarty, D. J., and Gatter, R. A. (1966). Recurrent acute inflammation associated with focal apatite crystal deposition. Arthritis and Rheumatism, 9, 804-819.

Meachim, G., and Emery, I. H. (1973). Cartilage fibrillation in shoulder and hip joints in Liverpool necropsies. Journal of Anatomy, 116, 161-179.

Muir, H. (1977). Molecular approach to the understanding of osteoarthrosis. Annals of the Rheumatic Diseases, 36, 199-208.

Murray, R. O., and Duncan, C. (1971). Athletic activity in adolescence as an aetiological factor in degenerative hip disease. Journal of Bone and Joint Surgery, 53B, 406-419.

Murray-Leslie, C. F., Lintott, D. J., and Wright, V. (1977). The knees and ankles in sport and veteran military parachutists. Annals of the Rheumatic Diseases, 36, 327-331.
Puranen, J., Ala-Ketola, L., Peltokallio, P., and Saarela, J. (1975). Running and primary osteoarthritis of the hip. British Medical Journal, 2, 424-425.

Radin, E. L. (1976). Aetiology of osteoarthrosis. Clinics in Rheumatic Diseases, 2, 509-522.

Radin, E. L., Paul, I. L., and Rose, R. M. (1972). Role of mechanical factors in pathogenesis of primary osteoarthritis. Lancet, 1, 519-522.

Reginato, A. J., Schumacher, H. R., and Martinez, V. A. (1974). The articular cartilage in familial chondrocalcinosis. Arthritis and Rheumatism, 17, 977-992.

Sokoloff, L. (1969). The Biology of Degenerative Joint Disease. University of Chicago Press, Chicago.

Vignon, E., Arlot, M., Meunier, P., and Vignon, G. (1974). Quantitative histological changes in osteoarthritic hip cartilage. Clinical Orthopaedics, 103, 269-278.

Weightman, B. (1975). In-vitro fatigue testing of articular cartilage. Annals of the Rheumatic Diseases, 34, Supp. 2, 108-110. 